\title{
NEUTRINO PROPAGATION IN NEUTRON MATTER AND THE NUCLEAR EQUATION OF STATE
}

\author{
J. MARGUERON \\ Groupe de Physique Théorique, IPN, F-91406 Orsay, France \\ J. NAVARRO \\ IFIC, Apdo. 22085, 46075 Valencia, Spain \\ N. VAN GIAI \\ Groupe de Physique Théorique, IPN, F-91406 Orsay, France \\ AND \\ W. JIANG \\ Institute of Nuclear Research, Shanghai 201800, China
}

\begin{abstract}
We study the propagation of neutrinos inside dense matter under the conditions prevailing in a proto-neutron star. Equations of state obtained with different nuclear effective interactions (Skyrme type and Gogny type) are first discussed. It is found that for many interactions, spin and/or isospin instabilities occur at densities larger than the saturation density of nuclear matter. From this study we select two representative interactions, SLy230b and D1P. We calculate the response functions in pure neutron matter where nuclear correlations are described at the Hartree-Fock plus RPA level. These response functions allow us to evaluate neutrino mean free paths corresponding to neutral current processes.
\end{abstract}

\section{Introduction}

Neutrinos play a crucial role during the energy liberation phase that follows a super-nova collapse as they carry away most of the initial gravitational energy. In order to describe the evolution of the newly born neutron star it is important to have reliable estimates of the mean free path of neutrinos in a medium with the typical characteristics of a proto-neutron star, namely with densities ranging between one and several times the value of the saturation density of symmetric nuclear matter, with temperatures of 
around a few tens of $\mathrm{MeV}$, and with a proton fraction which can represent up to $30 \%$ of the baryonic density.

We report here on some calculations of the mean free path of neutrinos in pure neutron matter under various conditions of density and temperature. The scattering of neutrinos on neutrons is mediated by the neutral current of the electroweak interaction. In the non-relativistic limit and in the case of non-degenerate neutrinos, the mean free path of a neutrino with initial momentum $\mathbf{k}_{1}$ is given by [1]

$$
\begin{aligned}
1 / \lambda\left(\mathrm{k}_{1}, T\right)=\frac{G_{F}^{2}}{16 \pi^{2}} \int d \mathbf{k}_{3}\left(c_{V}^{2}(1\right. & +\cos \theta) \mathcal{S}^{(0)}(q, T) \\
& \left.+c_{A}^{2}(3-\cos \theta) \mathcal{S}^{(1)}(q, T)\right)
\end{aligned}
$$

where $T$ is the temperature, $G_{F}$ is the Fermi constant, $c_{V}\left(c_{A}\right)$ the vector (axial) coupling constant, $\mathrm{k}_{3}$ the final neutrino momenta, $q=k_{1}-k_{3}$ the transferred energy-momentum, and $\cos \theta=\hat{\mathbf{k}}_{1} \cdot \hat{\mathbf{k}}_{3}$. The dynamical structure factors $\mathcal{S}^{(S)}(q, T)$ describe the response of neutron matter to excitations induced by neutrinos, and they contain the relevant information on the medium. The vector (axial) part of the neutral current gives rise to density (spin-density) fluctuations, corresponding to the $S=0(S=1)$ spin channel.

A microscopic framework based on effective nucleon-nucleon interactions is used here to describe consistently both the Equation of State (EOS) and the dynamical structure factors of neutron matter. Then, the mean free path of neutrinos is calculated according to Eq. (1) for different values of the density and temperature of the nuclear medium.

\section{The EOS and the choice of effective interactions}

Nucleon-nucleon effective interactions are usually fitted so as to reproduce some selected properties of finite nuclei and symmetric nuclear matter at saturation. The study of proto-neutron stars requires the use of these interactions for nuclear densities well beyond the saturation density of symmetric nuclear matter, and we explain in this section the criteria for our choice among the plethora of effective interactions currently in use. In Figure 1 is represented the energy per particle in symmetric nuclear matter and pure neutron matter as a function of the density $\rho$, as resulting from a mean field calculation employing several Skyrme and Gogny interactions. For comparison, the variational results given in [2] are also plotted; such results are based on the two realistic two-body interactions AV14 and UV14, plus the three-body interaction UVII. The noticeable differences between these two 

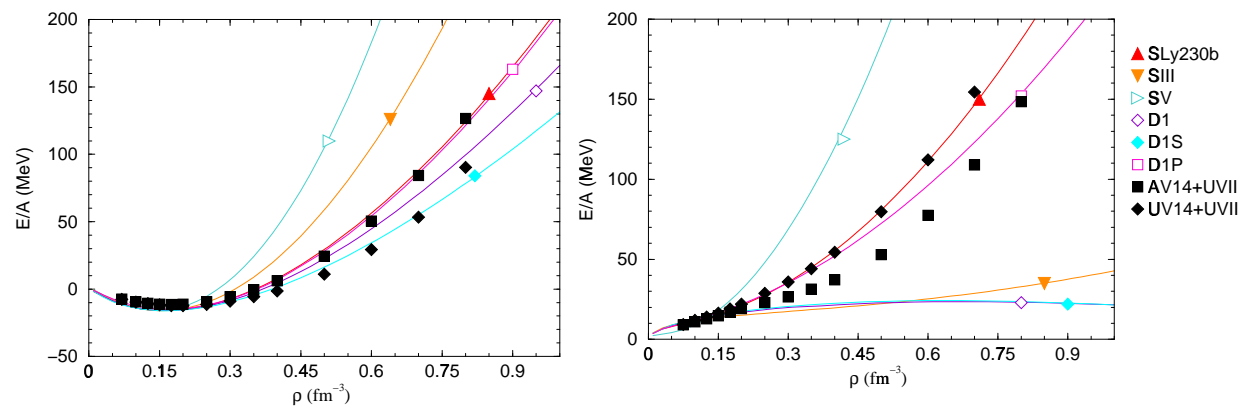

Figure 1. Energy per particle of symmetric nuclear matter (left panel) and neutron matter (right panel) as a function of density $\rho$ for a selected set of Skyrme and Gogny effective interactions. The black squares and diamonds are the results of variational calculations[2] based on realistic two- and three-body interactions.

upper bounds provide an estimate of the reliability of the calculations for densities beyond the saturation point.

In the left panel of Fig. 1 one can see that the Skyrme forces SIII and SV overestimate $E / A$ as compared with the variational results for values of the density larger than $0.25 \mathrm{fm}^{-3}$. Such a discrepancy has to be related to the high value of the compression modulus provided by these interactions $(\sim 350 \mathrm{MeV})$. The remaining effective interactions used in this figure give more reasonable values for the incompressibility (around $240 \mathrm{MeV}$ ), and one can see that their predicted energies per particle are in reasonable agreement with the variational results in the range of densities displayed in the figure. In contrast, in neutron matter (right panel) only the effective interactions SLy230b and D1P lead to energies per particle in agreement with the variational results. As a matter of fact, both SLy230 and D1P have included the variational results of neutron matter into the adjustment of parameters. Thus, we select two representative forces, one of Skyrme type (SLy230b[3]) and one of Gogny type (D1P[4]).

However, the agreement with the variational EOS is not the only criterium we have followed to select an effective interaction. We have also considered isospin and spin properties. The asymmetry energy is related to the second derivative of the energy per particle with respect to the asymmetry parameter $I=\left(\rho_{p}-\rho_{n}\right) / \rho$. For small values of $I$, one can write

$$
E(I) / A=E(0) / A+\epsilon_{1} I^{2}+O\left(I^{3}\right)
$$

where $\epsilon_{1}$ is the asymmetry energy coefficient. This coefficient must be positive to guarantee the stability of symmetric nuclear matter, otherwise a small fluctuation of $I$ would amplify leading to neutron matter as the more stable form of nuclear matter. In Figure2 is displayed the asymmetry energy $\epsilon_{1}$ as a function of the density for the same interactions used in Figure 


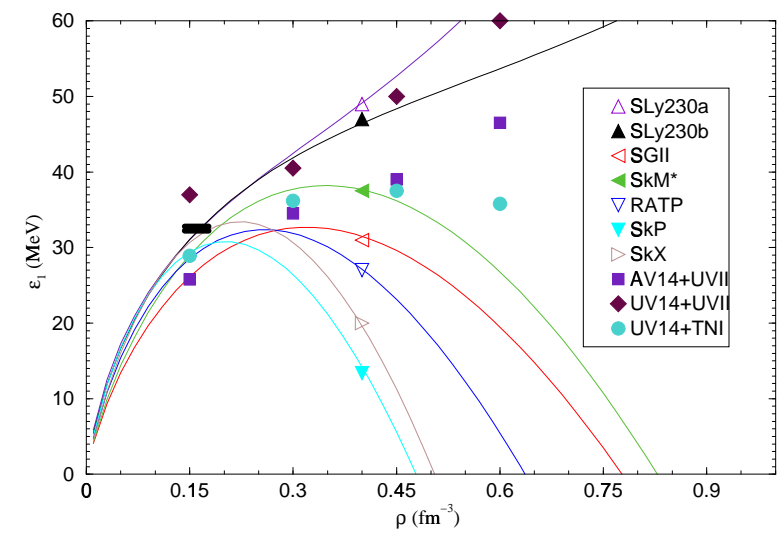

Figure 2. Asymmetry energy $\epsilon_{1}$ of nuclear matter as a function of density for a set of Skyrme and Gogny effective interactions. We also show the results of variational calculations[2]. The black rectangle represents the empirical point including error bars.

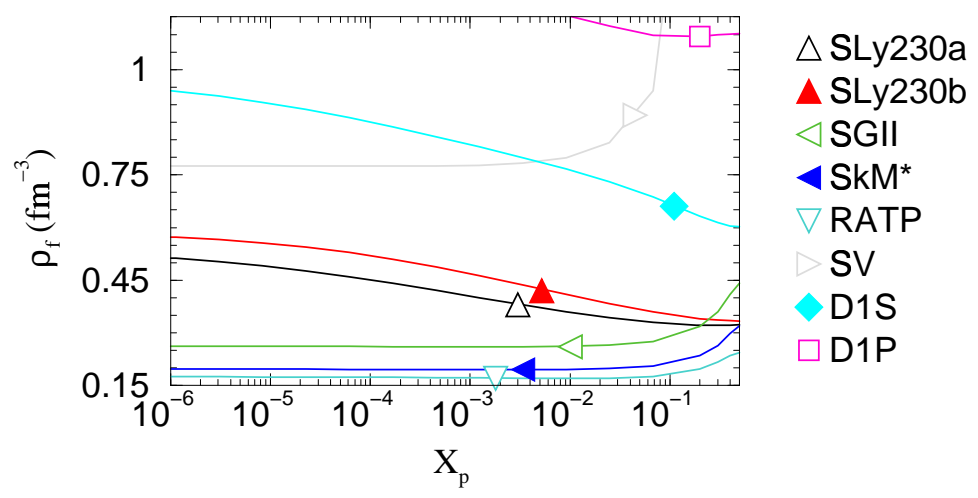

Figure 3. Ferromagnetic density as a function of the proton fraction $X_{p}$ for a selected set of Skyrme and Gogny effective interactions.

1. The value of $\epsilon_{1}$ as deduced from the semi-empirical mass formula ( $\epsilon \simeq 32$ $\mathrm{MeV}$ at saturation, indicated in Figure 2 as a small black rectangle) is reproduced by most of the effective interactions. Apart from the parametrization SLy230, the displayed Skyrme interactions lead to an isospin instability for densities a few times higher than the saturation density. The onset of such an instability is the value of the density at which $\epsilon_{1}$ becomes zero.

We have also looked at the spin properties of the effective interactions. In particular, we have calculated the magnetic susceptibility of asymmetric nuclear matter [5]. In Figure 3 is plotted as a function of the proton fraction $X_{p}=\rho_{p} / \rho$ the value of the ferromagnetic density, which is defined as the density at which a ferromagnetic instability appears. It can be seen that all 


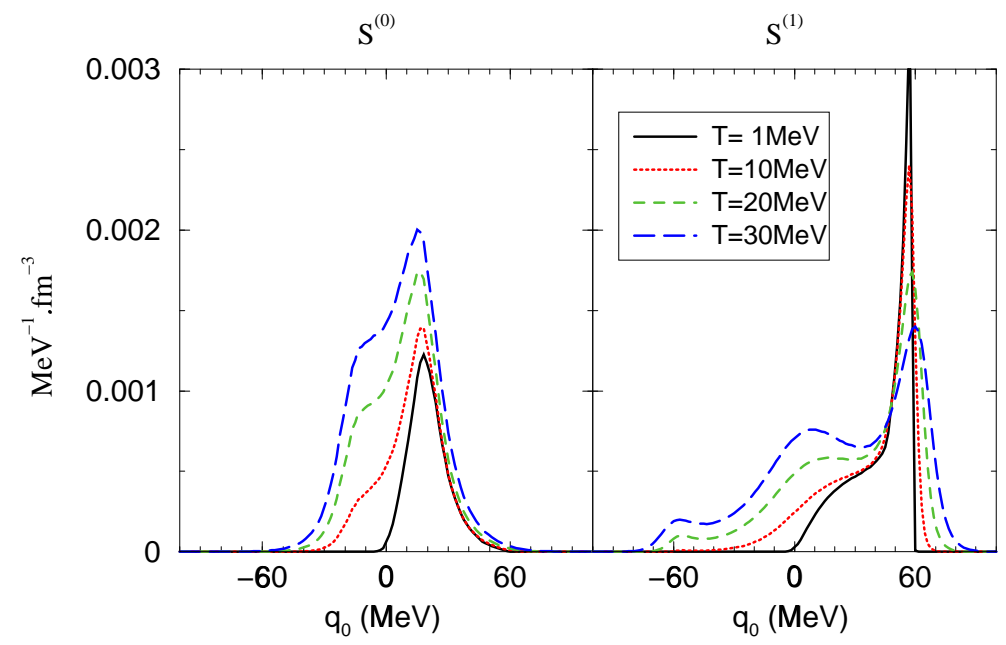

Figure 4. RPA dynamical structure factors $\mathcal{S}^{(S)}(q, T)$ for neutron matter as a function of transferred energy $q_{0}$. Calculations have been done at the saturation density of symmetric nuclear matter, for a transferred momentum $\mathrm{q}=k_{F} / 3$ and for several temperatures. The interaction is SLy230b.

Skyrme interactions predicts such an instability in the range of densities of relevance for the study of a proto-neutron star. Note that the case of pure neutron matter corresponds to $X_{p}=0$, at the left of the figure. In contrast, Gogny-type interactions predict a ferromagnetic instability for densities which are well beyond the range of values where the mean field plus effective interactions can be reasonably used.

In conclusion, we shall employ the interactions SLy230b and D1P for our calculations of the dynamical structure factors in neutron matter.

\section{Dynamical structure factors}

The detailed balance relation allows one to obtain the dynamical structure factors $\mathcal{S}^{(S)}$ from the imaginary part of the response function $\chi^{(S)}(q, T)$ in pure neutron matter as

$$
\mathcal{S}^{(S)}(q, T)=-\frac{1}{\pi} \frac{1}{1-\exp \left(-\mathrm{q}_{0} / T\right)} \Im \mathrm{m} \chi^{(S)}(q, T) .
$$

We recall that $q$ is a four-vector $\left(q_{0}, \mathbf{q}\right)$, and this expression is valid for both positive and negative transferred energies $q_{0}$.

For Skyrme type interactions, the response function can be exactly calculated including the full RPA correlations (see [6] and references therein). The expressions for pure neutron matter are given in [6] (note that, in [6] Eq.(20) should be multiplied by a factor $\left.\left(1-e^{-\beta \omega}\right) / 2\right)$. In Figure 4 are 
displayed the dynamical structure factors for the density $(S=0)$ and spindensity $(S=1)$ channels as a function of the transferred energy qo from the probe to the medium. At zero temperature, only positive values of the transferred energy are accessible, which accounts for the heating of the medium by the probe. As the temperature increases, the structure factors grow in the negative transferred energy domain. This behaviour corresponds to the cooling of the medium by the probe. The pronounced zero sound in the spin-density channel at zero temperature is damped when the temperature increases. As a general rule, when the value of the density increases and approaches the ferromagnetic point mentioned in the previous section, a dramatic peak appears in the structure factor $[6,5]$.

We have also calculated the response function assuming several interesting approximations for the particle-hole effective interaction. For a free Fermi gas the response function is given by the well-known Lindhard function. The effects of the interaction at the mean-field or Hartree-Fock level imply the use of an effective mass in the Lindhard function. This is exact for Skyrme-Hartree-Fock and represents a convenient approximation in the case of Gogny-Hartree-Fock[5]. The particle-hole interaction may be also approached by a Landau form, containing only $l=0$ multipole, or $l=0,1$ multipoles. We stress that in all cases the particle-hole interaction has been consistently obtained from the particle-particle interaction used to describe the EOS. The full RPA response function has been exactly calculated for Skyrme interactions. For the Gogny interaction we have used the effective mass approximation for the Hartree-Fock response and the Landau approximation for the RPA response.

\section{Neutrino mean free paths}

The temperature dependence of the neutrino mean free path can be seen in Figure 5, where the calculated $\lambda$ has been represented in the case of a free Fermi gas, and in the mean field approximation using SLy230b and D1P interactions. We have chosen an incoming neutrino energy $E_{1}=60 \mathrm{MeV}$. Only at very low temperatures the behavior of $\lambda$ follows the expected law in $T^{-2}$ [7] (indicated also in the figure). As the temperature increases the interaction effects, contained in the effective mass, become less important and one reaches the free Fermi gas value at very high temperatures.

The effects of the residual interaction, i.e., of RPA correlations, can be seen in Figure 6, where we have represented the ratio of RPA to HartreeFock mean free paths as a function of the density. For SLy230b, we have calculated the response function using either the full particle-hole interaction (labeled RPA in the figure) or its Landau approximation (labeled RPA-Landau in the figure). Only the latter approximation has been used 


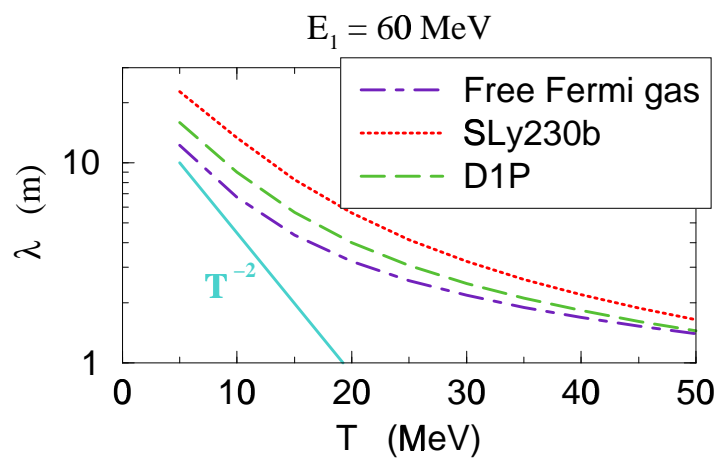

Figure 5. Mean free path in neutron matter as a function of the temperature. The curves labeled SLy230b and D1P have been obtained within the mean field approximation for the response function. The energy of the incident neutrino is $E_{1}=60 \mathrm{MeV}$.

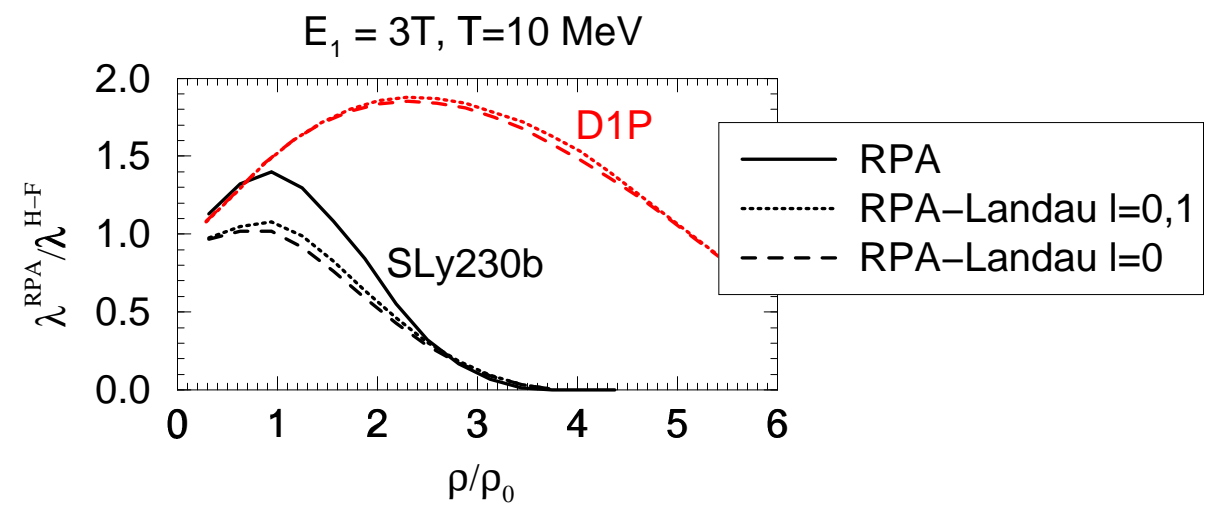

Figure 6. The relative mean free path $\lambda^{\mathrm{RPA}} / \lambda^{\mathrm{HF}}$ as a function of the density for interactions SLy230b and D1P.

for the interaction D1P.

In the case of interaction SLy230b one can see that the effects of the residual interaction in Landau approximation (either $l=0$ or $l=0,1$ ) do not produce any significant difference as compared to the HF approximation for densities around the saturation value. In the same region, the full residual interaction produces a sizeable increase of $\lambda$. All three solutions predict a strong decrease of the mean free path when the density reaches the ferromagnetic density. This is due to the very strong axial response function as the system comes closer to the ferromagnetic instability. In contrast, for interaction D1P a significant difference with respect to the HF results appears when the residual interaction is considered at the Landau level. Note that the differences in the results obtained using the Landau approx- 
imations with $l=0$ or with $l=0,1$ are quite small. Finally, the force D1P also predicts a vanishing mean free path when the density increases, but the ferromagnetic instability happens at very high densities, well beyond the domain of applicability of the effective interaction approach.

\section{Conclusions}

In the domain of densities and protonic fractions that are relevant to the proto-neutron star evolution, the use of effective nucleon-nucleon interactions which are originally designed for describing the neighbourhood of normal nuclear matter is a delicate issue. These interactions may fail to reproduce the EOS of neutron matter (SIII. SV, D1, D1S), or they lead to isospin and/or spin instabilities at 2-3 times normal density (Skyrme forces).

Having this in mind, it is still possible to use some of the existing effective interactions to calculate consistently the nuclear response functions and hence the neutrino mean free paths in neutron matter. The evolution of the response functions with increasing temperatures shows that neutrinos can efficiently mediate the cooling process of hot neutron matter. We find that RPA correlations have a significant effect on mean free paths. With Skyrme interactions, RPA correlations tend to increase mean free paths at densities around normal density whereas at larger densities they predict very short mean free paths because of the onset of spin instabilities. Thus, these interactions do not favor a fast cooling process. On the other hand, the Gogny interaction predicts a quite different scenario where RPA correlations increase mean free paths in the whole range of densities (up to about $\left.5 \rho_{0}\right)$. In this latter case where ferromagnetic instabilities are not present, the mean field and the RPA correlations make the nuclear matter more transparent compared to the free Fermi gas model. Thus, neutrinos would escape more quickly and the delayed mecanism will be less efficient.

\section{References}

1. N. Iwamoto and C.J. Pethick, Phys. Rev. D 25 (1982) 313.

2. R.B. Wiringa, V.Fiks and A.Fabrocini, Phys. Rev. C 38 (1988) 1010; R.B. Wiringa, Rev. Mod. Phys. 65 (1993) 231.

3. E. Chabanat, P. Bonche, P, Haensel, J. Meyer and R. Schaeffer, Nucl. Phys. A627 (1997) 710.

4. M.Farine, D.Van-Eiff, P.Schuck, J.-F.Berger, J.Dechargé, and M.Girod, J.Phys.G : Nucl.Part.Phys. 25 (1999) 863.

5. J.Margueron, PhD thesis (2001) Paris, unpublished.

6. J. Navarro, E.S. Hernández and D. Vautherin, Phys. Rev. C 60 (1999) 045801.

7. S. Reddy, M. Prakash and J.M.Lattimer, Phys. Rev. D 58 (1998) 013009. 